\title{
Scanning fiber endoscopy: multimodes of guided intervention (Presentation Video)
}

\section{Eric Seibel}

Eric J. Seibel, "Scanning fiber endoscopy: multimodes of guided intervention (Presentation Video)," Proc. SPIE 8927, Endoscopic Microscopy IX; and Optical Techniques in Pulmonary Medicine, 89271P (21 May 2014); doi: 10.1117/12.2072698

\section{SPIE. Event: SPIE BiOS, 2014, San Francisco, California, United States}




\section{Scanning fiber endoscopy: multimodes of guided intervention (Presentation Video)}

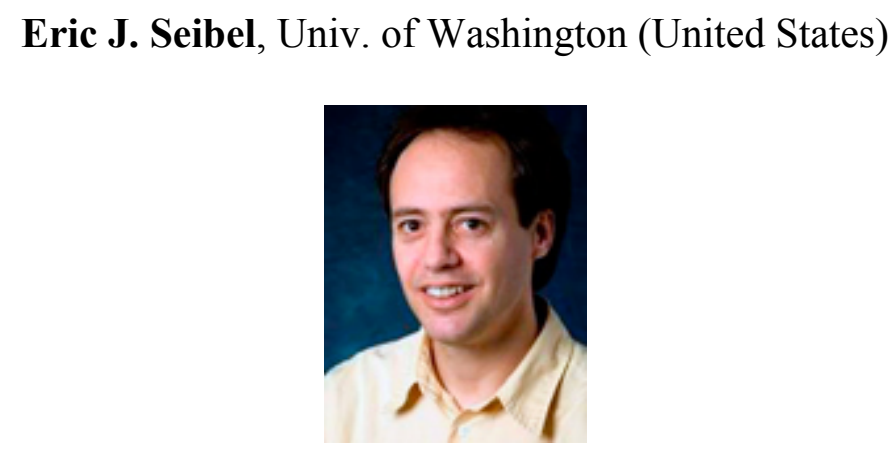

\section{BIOS 2014 Hot Topics Presentation}

In this Hot Topics presentation,Eric Seibel discusses his team's work using a scanning-fiber endoscope (SFE) to provide high-contrast imaging. The instrument consists of red-green-blue illumination sources coupled into a single-mode fiber optic that can be scanned in 2D using a piezoelectric tube as an actuator. The output end of the device consists of a quartz cantilever, an asphere lens, and fluorescence detectors coupling high-resolution spectral imaging with a long depth of focus.

Compared to current state-of-the-art instruments such as a tethered capsule endoscope with outer diameters on the order of $6 \mathrm{~mm}$, the SFE has an outer diameter of only $1.2 \mathrm{~mm}$ making it suitable for procedures in small ducts and the cardiovascular system. Seibel discusses using the instrument for improving biopsy procedures and diagnostics as well as in stent deployment.

View presentation video on SPIE's Digital Library:

http://dx.doi.org/10.1117/12.2072698.3578057222001 